BOVI, M.L.A.; MARTINS, C.C.; SPIERING, S.H. Desidratação de sementes de quatro lotes de pupunheira: efeitos sobre a germinação e o vigor. Horticultura Brasileira, Brasília, v. 22, n. 1, p. 109-112, jan-mar 2004.

\title{
Desidratação de sementes de quatro lotes de pupunheira: efeitos sobre a germinação e o vigor
}

\author{
Marilene L.A. Bovi ${ }^{1}$; Cibele Chalita Martins ${ }^{2}$; Sandra Heiden Spiering ${ }^{1}$

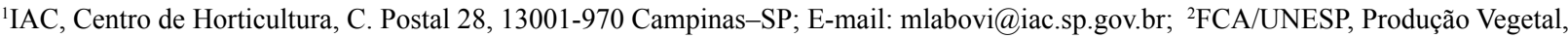 \\ C. Postal 237, 18603-970 Botucatu-SP
}

\section{RESUMO}

Com o objetivo de verificar o efeito da desidratação sobre a germinação e o vigor de sementes de pupunheira inerme (Bactris gasipaes Kunth), quatro lotes de sementes, colhidos nas localidades de Yurimaguas, Peru; Camamu e Piraí do Norte, BA e Pindorama, SP, foram submetidos à secagem em câmara seca a partir das testemunhas não desidratadas, retirando-se amostras a cada 24 horas. $\mathrm{O}$ efeito da desidratação sobre a qualidade das sementes foi avaliado por meio de teste de germinação (184 dias da semeadura, em vermiculita, $20-30^{\circ} \mathrm{C}$ ), primeira contagem de germinação (36 dias), velocidade de germinação e teor de água das sementes. Comprovou-se que sementes dessa espécie são sensíveis à desidratação, com germinação inicial alta (59 a 84\%) quando não desidratadas (47 a $38 \%$ de umidade inicial). Teores de água abaixo da faixa de 28 a $23 \%$ reduziram significativamente a germinação e o vigor. Todas as sementes com teores de umidade abaixo de $15 \%$ (lotes 2 e 4 ) e $13 \%$ (lotes 1 e 3) morreram.

Palavras chave: Bactris gasipaes, deterioração da semente, recalcitrante, teor de água crítico, teor de água letal.

\begin{abstract}
Dehydration effects on germination and vigor of four pejibaye seed lots

The dessication tolerance of four seed lots of spineless pejibaye (Bactris gasipaes Kunth) harvested at Yurimaguas (Peru); Camamu and Piraí do Norte (Bahia State, Brazil) and Pindorama (São Paulo State, Brazil) was identified. The seeds were dried in a drying chamber starting with undried controls. Samples were drawn every 24 hours. Dessication effects were evaluated by means of germination test (until 184 days, vermiculite, $20-30^{\circ} \mathrm{C}$ ), germination first counting (36 days), germination speed and seed water content. It was verified that seeds from that species are susceptible to dehydration, with higher initial germination percentage (59 to $84 \%$ ) when undried (47 to $38 \%$ of water content). Water contents lower than 28 to $23 \%$ reduced significantly seed germination and vigor. All seeds died when the seed water content was lower than $15 \%$ (seed lots 2 and 4 ) and $13 \%$ (seed lots 1 and 3 ).
\end{abstract}

Keywords: Bactris gasipaes, peach palm, seed deterioration, recalcitrance, critical water content, lethal water content.

(Recebido para publicação em 09 de abril de 2003 e aceito em 10 de outubro de 2003)

\begin{abstract}
A pupunheira (Bactris gasipaes) é espécie produtora de palmito, hortaliça não convencional, largamente cultivada no Brasil e em vários países da América Latina. A expansão da área de cultivo, realizada a partir da década de 90 , fez crescer a procura por sementes e mudas dessa palmeira, particularmente sobre ecótipos inermes, que por não apresentarem espinhos, facilitam os tratos culturais. No entanto, os conhecimentos sobre a fisiologia da germinação dessa espécie são ainda limitados, sendo essenciais para o desenvolvimento de tecnologias favoráveis à produção e à conservação de suas sementes.
\end{abstract}

Sementes de Bactris gasipaes foram classificadas como recalcitrantes (Ferreira e Santos, 1992). As sementes recalcitrantes apresentam teores de água definidos como críticos, abaixo dos quais a viabilidade é reduzida. Apresentam também, teores de água letais, relacionados à perda total da viabilidade (Probert e Longley, 1989; Hong e Ellis,
1992). O conhecimento dos teores de água crítico e letal de uma espécie é indispensável para o planejamento e a execução da secagem e do armazenamento das sementes, pois o teor de água é um fator determinante do comportamento das sementes recalcitrantes. Nessas sementes, a água sub-celular está fortemente associada às superfícies macromoleculares assegurando, em parte, a estabilidade de membranas e macromoléculas. A perda de água estrutural durante o processo de secagem causaria a alteração de sistemas metabólicos e de membranas, resultando no início do processo de deterioração (Farrant et al., 1988).

Em estudos com lotes de sementes provenientes de diferentes locais ou de ecótipos de uma mesma espécie é considerada a existência de uma faixa, e não de um valor único, para teores de água crítico e letal. Isso ocorre devido a sensibilidades diferenciadas na tolerância à perda de água observadas dentro de uma mesma espécie (Ellis et al., 1991; Martins et al., 1999 abc), de acordo com o habitat de origem, condições agrobioclimáticas de cultivo e a diversidade genética (Farrant et al., 1988; Martins et al., 1999 abc).

O objetivo do estudo foi verificar o efeito da desidratação de sementes de pupunheiras inermes sobre a germinação e o vigor.

\section{MATERIAL E MÉTODOS}

Os frutos foram colhidos em Yurimaguas, Peru; Camamu e Piraí do Norte, BA e Pindorama, SP, em plantas matrizes selecionadas para vigor e ausência de espinhos. Tão logo colhidos, os frutos foram despolpados, e as sementes lavadas e enviadas para Campinas. Cada lote de sementes foi dividido em nove sub-lotes, de peso similar. Um dos sub-lotes foi mantido sem secagem, constituindo o tratamento testemunha e os demais sub-lotes foram colocados em 


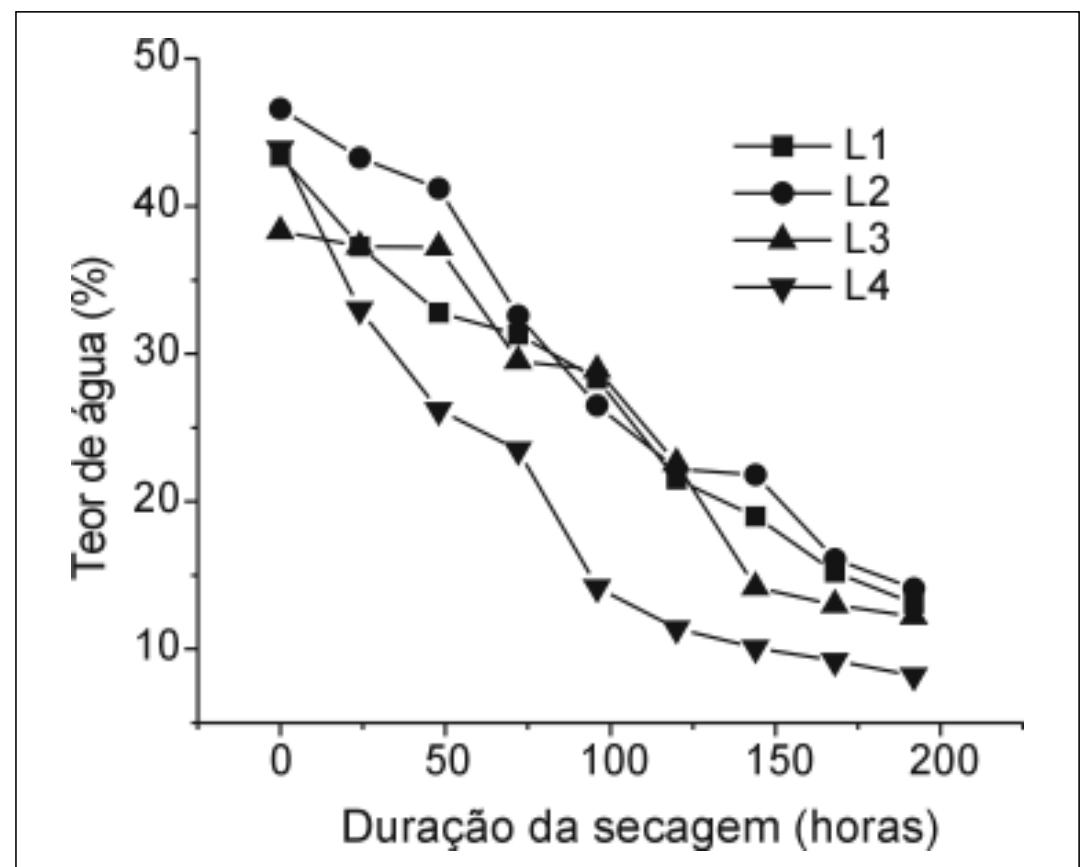

Figura 1. Curvas de secagem dos quatro lotes de pupunheira. L1 - Yurimaguas, Peru; L2 Camamu, BA; L3 - Piraí do Norte, BA; L4 - Pindorama, SP. Campinas, IAC, 2001.

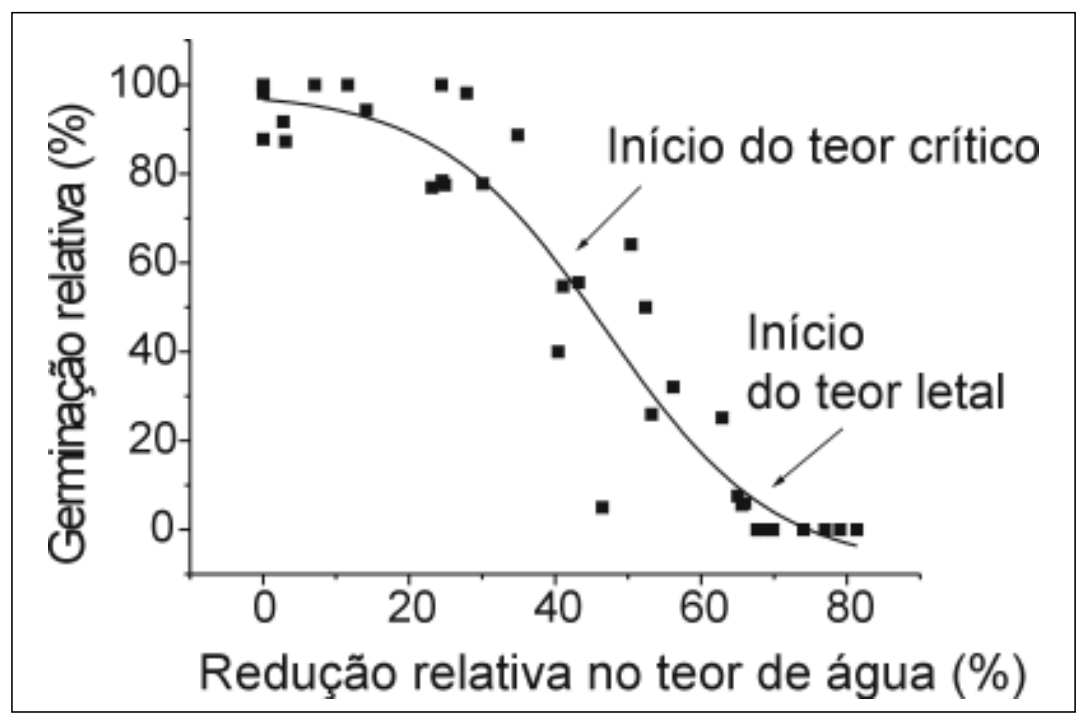

Figura 2. Germinação média relativa de quatro lotes de pupunheira em função da redução média do teor de água das sementes. Campinas, IAC, 2001.

bandejas dentro de câmara seca, retirando-se um sub-lote a cada 24 horas de secagem. Nesse ambiente, a temperatura e a umidade relativa do ar foram $28,5 \pm 2,5^{\circ} \mathrm{C}$ e $40 \pm 10 \%$, respectivamente. A determinação do teor de água das sementes foi realizada antes e após os diversos períodos de secagem, utilizando-se o método da estufa a $105 \pm 3^{\circ} \mathrm{C}$ por 24 horas, e três subamostras de três sementes inteiras. $\mathrm{O}$ efeito da desidratação foi avaliado por meio de teste de germinação, utilizando-se quatro subamostras de 20 sementes por tratamento, em vermiculita, a $20-30^{\circ} \mathrm{C}$ e luz (78 $\mu \mathrm{mol} \mathrm{s}^{-1} \mathrm{~m}^{-2}$ por 8 horas), contabilizando-se as plântulas normais emersas 184 dias após a semeadura.

Como medidas do vigor, foi utilizada a primeira contagem (efetuada aos
36 dias após a semeadura) e o índice de velocidade de germinação, contabilizando-se, semanalmente, até 184 dias da semeadura, as plântulas emersas e adaptando-se o critério estabelecido por Maguire (1962).

$\mathrm{Na}$ avaliação, foi considerado como teor de água crítico aquele a partir do qual houve redução significativa na porcentagem de germinação. Considerouse como teor de água letal, aquele a partir do qual não houve germinação das sementes (Hong e Ellis, 1992).

O delineamento experimental utilizado foi o inteiramente casualizado com os tratamentos em quatro repetições. Foi efetuada a análise de variância, aplicando-se o teste $\mathrm{F}$ e comparando-se médias dos tratamentos pelo teste Tukey (5\%). A análise conjunta dos dados relativos dos quatro lotes foi realizada via regressão e ajuste de curva, utilizando-se o modelo sigmoidal de Boltzman.

\section{RESULTADOS E DISCUSSÃO}

A Figura 1 mostra a relação entre o teor de água das sementes e o tempo de secagem, obtida pelo método utilizado. O processo de secagem adotado pode ser classificado como lento (Pammenter et al., 1998), pois demandou 192 horas para que o teor de água inicial das sementes (47 a $38 \%$ ) baixasse para 14 a $8 \%$. A desidratação progressiva intensificou o processo de deterioração, como verificado na porcentagem média relativa de germinação (Figura 2) e também na análise, por lotes, da distribuição da germinação ao longo do tempo e do índice de velocidade de germinação (Tabela 1), confirmando o comportamento recalcitrante das sementes de Bactris gasipaes, anteriormente reportado por Ferreira e Santos (1992) para o ecótipo mesocarpa com espinhos. Para cada lote de sementes estudado, observa-se que antes mesmo de ser atingido o teor de água crítico, quanto menor o teor de água das sementes, maior o tempo necessário para a germinação (Tabela 1).

A deterioração causada pela desidratação das sementes de palmeiras, como Euterpe edulis, E. espiritosantensis e E. oleracea (Martins et al., 1999 abc) e Chrysalidocarpus lutescens (Becwar et al., 1982) afeta, também, o vigor, dani- 
Tabela 1. Médias dos parâmetros utilizados na avaliação dos teores crítico e letal de água de quatro lotes de sementes de Bactris gasipaes. Campinas, IAC, 2001.

\begin{tabular}{|c|c|c|c|c|c|c|c|c|c|}
\hline \multirow[b]{2}{*}{ Lotes } & \multirow{2}{*}{\multicolumn{2}{|c|}{ Teor de água }} & \multicolumn{6}{|c|}{ Germinação (dias após a semeadura) } & \multirow{3}{*}{ IVG } \\
\hline & & & 36 & 45 & 66 & 87 & 124 & 184 & \\
\hline & \multicolumn{8}{|c|}{ (\%) } & \\
\hline \multirow[t]{9}{*}{1} & 43,4 & & 46,25 a & $50,62 a$ & $55,00 a$ & $63,75 a$ & $65,00 a$ & $65,00 a$ & $1,59 a$ \\
\hline & 37,3 & & $28,75 a b$ & $41,25 a b$ & $53,75 a b$ & $58,75 a b$ & $62,50 a b$ & $66,25 a$ & $1,67 a$ \\
\hline & 32,8 & & $22,50 \mathrm{bc}$ & $35,62 a b c$ & $48,75 a b c$ & $57,50 a b$ & $62,50 a b$ & $62,50 \mathrm{ab}$ & $1,49 a$ \\
\hline & 31,3 & & $13,75 \mathrm{bcd}$ & $25,00 \mathrm{bc}$ & $36,25 \mathrm{~cd}$ & $55,00 a b$ & $63,75 a b$ & $65,00 a$ & $1,27 a$ \\
\hline & 28,3 & & $5,00 \mathrm{~cd}$ & $18,75 \mathrm{~cd}$ & $32,50 \mathrm{c}$ & $50,00 a b$ & $55,00 a b$ & $58,75 a b$ & $1,11 \mathrm{a}$ \\
\hline & 21,5 & Crítico & $0,00 \mathrm{~d}$ & $5,00 \mathrm{de}$ & $10,00 d$ & $41,25 b$ & $42,50 b$ & $42,50 \mathrm{bc}$ & $0,34 b$ \\
\hline & 19,0 & & $0,00 d$ & $2,50 \mathrm{de}$ & $5,00 d$ & $18,75 c$ & $20,00 \mathrm{c}$ & $21,25 \mathrm{~cd}$ & $0,33 b$ \\
\hline & 15,2 & & $0,00 d$ & $0,00 \mathrm{e}$ & $0,00 d$ & $0,00 \mathrm{c}$ & $0,00 \mathrm{c}$ & $5,00 d$ & $0,17 b$ \\
\hline & 13,1 & Letal & $0,00 \mathrm{~d}$ & $0,00 \mathrm{e}$ & $0,00 \mathrm{~d}$ & $0,00 \mathrm{c}$ & $0,00 \mathrm{c}$ & $0,00 \mathrm{~d}$ & $0,00 \mathrm{~b}$ \\
\hline \multirow[t]{9}{*}{2} & 46,6 & & $0,00 a$ & $7,00 \mathrm{a}$ & $22,00 a b$ & $37,00 a b c$ & $59,00 a b$ & $59,25 a b$ & $1,72 a$ \\
\hline & 43,3 & & $0,00 a$ & $3,75 a b$ & $30,00 a$ & $56,25 a$ & $67,50 a$ & $67,50 a$ & $1,09 a b$ \\
\hline & 41,2 & & $0,00 a$ & $1,25 b$ & $25,62 a b$ & $50,00 a b$ & $67,50 a$ & $67,50 a$ & $0,62 b c$ \\
\hline & 32,6 & & $0,00 a$ & $0,00 \mathrm{~b}$ & $16,25 b c$ & $32,50 \mathrm{bc}$ & $47,50 a b c$ & $52,50 a b$ & $0,28 \mathrm{bc}$ \\
\hline & 26,5 & & $0,00 a$ & $0,00 \mathrm{~b}$ & $11,25 \mathrm{~cd}$ & $22,50 \mathrm{~cd}$ & $36,25 b c$ & $37,50 \mathrm{bc}$ & $0,22 b c$ \\
\hline & 22,2 & & $0,00 a$ & $0,00 \mathrm{~b}$ & $5,62 \mathrm{de}$ & $11,25 \mathrm{de}$ & $31,25 \mathrm{~cd}$ & $33,75 b c$ & $0,21 b c$ \\
\hline & 21,8 & Crítico & $0,00 a$ & $0,00 \mathrm{~b}$ & $3,75 \mathrm{de}$ & 7,50de & $15,00 \mathrm{de}$ & $17,50 \mathrm{~cd}$ & $0,10 \mathrm{bc}$ \\
\hline & 16,1 & & $0,00 a$ & $0,00 \mathrm{~b}$ & $0,00 \mathrm{e}$ & $0,00 \mathrm{e}$ & $2,50 \mathrm{e}$ & $3,75 d$ & $0,02 c$ \\
\hline & 14,1 & Letal & $0,00 a$ & $0,00 \mathrm{~b}$ & $0,00 \mathrm{e}$ & $0,00 \mathrm{e}$ & $0,00 \mathrm{e}$ & $0,00 d$ & $0,00 c$ \\
\hline \multirow[t]{9}{*}{3} & 38,3 & & $0,00 a$ & $5,00 a$ & $22,75 a b$ & $40,45 a b c$ & $80,75 a$ & $84,50 a$ & $2,56 a$ \\
\hline & 37,3 & & $0,00 a$ & $5,00 a$ & $33,12 a$ & $61,25 a$ & $72,50 \mathrm{ab}$ & $77,50 a$ & $1,37 b$ \\
\hline & 37,2 & & $0,00 a$ & $0,00 a$ & $23,12 a b$ & $46,25 a b$ & $70,00 a b$ & $73,75 a b$ & $0,41 b c$ \\
\hline & 29,5 & & $0,00 a$ & $2,50 a$ & $15,00 \mathrm{bc}$ & $27,50 \mathrm{bcd}$ & $62,50 \mathrm{ab}$ & $65,00 \mathrm{ab}$ & $0,89 b c$ \\
\hline & 28,9 & & $0,00 a$ & $0,00 a$ & $17,50 \mathrm{~b}$ & $35,00 \mathrm{bc}$ & $60,00 a b$ & $66,25 a b$ & $0,38 b c$ \\
\hline & 22,6 & Crítico & $0,00 a$ & $0,00 a$ & $10,62 \mathrm{bcd}$ & $21,25 \mathrm{cde}$ & $48,75 b$ & $48,75 b c$ & $0,31 b c$ \\
\hline & 14,2 & & $0,00 a$ & $0,00 \mathrm{a}$ & $2,50 \mathrm{~cd}$ & $5,00 \mathrm{de}$ & $17,50 \mathrm{c}$ & $21,25 \mathrm{~cd}$ & $0,13 c$ \\
\hline & 13,0 & & $0,00 a$ & $0,00 a$ & $0,62 d$ & $1,25 \mathrm{e}$ & $3,75 \mathrm{c}$ & $5,00 \mathrm{~d}$ & $0,03 c$ \\
\hline & 12,2 & Letal & $0,00 a$ & $0,00 a$ & $0,00 d$ & $0,00 \mathrm{e}$ & $0,00 c$ & $0,00 d$ & $0,00 \mathrm{c}$ \\
\hline \multirow[t]{9}{*}{4} & 43,9 & & $0,00 a$ & $0,00 a$ & $30,00 a$ & $46,67 a$ & $66,67 a$ & $66,67 a$ & $3,65 a b$ \\
\hline & 33,0 & & $0,00 a$ & $0,00 a$ & $25,00 a b$ & $38,33 a$ & $46,67 a b$ & $51,67 a b$ & $2,79 a b$ \\
\hline & 26,2 & Crítico & $0,00 a$ & $0,00 a$ & $8,33 \mathrm{bc}$ & $16,67 \mathrm{~b}$ & $26,67 \mathrm{bc}$ & $26,67 b c$ & $1,35 b c$ \\
\hline & 23,5 & & $0,00 a$ & $0,00 a$ & $0,00 c$ & $1,67 b$ & $1,67 c$ & $1,67 \mathrm{c}$ & $0,14 \mathrm{c}$ \\
\hline & 14,2 & Letal & $0,00 a$ & $0,00 a$ & $0,00 \mathrm{C}$ & $0,00 b$ & $0,00 c$ & $0,00 c$ & $0,00 c$ \\
\hline & 11,4 & & $0,00 a$ & $0,00 a$ & $0,00 \mathrm{C}$ & $0,00 b$ & $0,00 c$ & $0,00 c$ & $0,00 c$ \\
\hline & 10,1 & & $0,00 a$ & $0,00 a$ & $0,00 \mathrm{C}$ & $0,00 \mathrm{~b}$ & $0,00 \mathrm{c}$ & $0,00 \mathrm{c}$ & $0,00 \mathrm{c}$ \\
\hline & 9,2 & & $0,00 a$ & $0,00 a$ & $0,00 \mathrm{C}$ & $0,00 \mathrm{~b}$ & $0,00 c$ & $0,00 c$ & $0,00 c$ \\
\hline & 8,2 & & $0,00 a$ & $0,00 a$ & $0,00 \mathrm{C}$ & $0,00 \mathrm{~b}$ & $0,00 \mathrm{c}$ & $0,00 \mathrm{c}$ & $0,00 \mathrm{c}$ \\
\hline
\end{tabular}

Médias do mesmo lote, seguidas pela mesma letra na coluna não diferem entre si pelo teste de Tukey a 5\%. Lote 1 - Yurimaguas, Peru; Lote 2 - Camamu, BA; Lote 3 - Piraí do Norte, BA; Lote 4 - Pindorama, SP.

ficando as membranas celulares, tornando a germinação mais lenta e diminuindo o crescimento das estruturas das plântulas (Becwar et al., 1982; Martins et al., $1999 \mathrm{abc})$. Isso faz com que sementes e plântulas fiquem mais vulneráveis e sujeitas, por mais tempo, às condições adversas do meio.
Em sementes recalcitrantes, a água sub-celular está fortemente associada às superfícies macromoleculares, assegurando em parte, a estabilidade de 
membranas e macromoléculas. Farrant et al. (1988) esclarecem que a perda de água estrutural durante o processo de secagem de sementes recalcitrantes pode causar severas alterações dos sistemas metabólicos e de membranas, dando início ao processo de deterioração dessas sementes.

Os lotes estudados apresentaram qualidades fisiológicas distintas, com diferenças significativas de teor de água inicial, germinação e índice de velocidade de germinação. Considerando esses fatores, a Tabela 1 mostra que para as sementes não desidratadas, o lote 2 , com maior teor de água $(46,6 \%)$, foi o que apresentou menor porcentagem final de germinação $(59,2 \%)$ e o lote 3 , com menor teor de água (38,3\%), foi o que apresentou maior porcentagem final de germinação. Os lotes 1 e 4, por sua vez, mantiveram-se numa categoria intermediária quanto ao teor de água (43,4 e 43,9\%, respectivamente) e germinação inicial $(65,0$ e $66,7 \%$, respectivamente). Porcentagem final de germinação entre 95 e $40 \%$ foi reportado anteriormente para 373 lotes de pupunheiras inermes da mesma localidade (Bovi et al., 1994). Para sementes provenientes de germoplasma com espinhos alguns autores relataram porcentagem final de germinação variando de 64,4 a 86,3\% (Coates-Beckford e Chung, 1987; Carvalho e Müller, 1998).

Para todos os lotes, a secagem das sementes até teores de água inferiores a valores entre 23 a $28 \%$ reduziu significativamente a porcentagem de germinação e o vigor, caracterizando uma faixa de teor de água crítico para a espécie (Tabela 1). A mortalidade total das sementes foi observada com a desidratação até teores de água abaixo de 13 a $15 \%$, dependendo do lote, caracterizando a faixa de teor de água letal para a pupunheira.

A faixa de teor de água crítico obtida no presente trabalho apresenta valores inferiores aos observados por Ferreira e Santos (1992) e Carvalho e
Müller (1998) para sementes da mesma espécie.

Por sua vez a faixa de teor letal é superior à descrita por Carvalho e Müller (1998) e inferior à relatada por Ferreira e Santos (1992).

Há provavelmente, sensibilidade diferenciada na tolerância à perda de água entre ecótipos de origem diferente dentro de uma mesma espécie (Farrant et al., 1988; Ellis et al., 1991; Martins et al., 1999ab). No presente trabalho foram utilizadas sementes do ecotipo inerme proveniente originalmente de Yurimaguas, material genético responsável atualmente por acima de $95 \%$ da pupunha cultivada no Brasil (Bovi, 1998). Por sua vez, as sementes utilizadas por Ferreira e Santos (1992) e também por Carvalho e Müller (1998) foram provenientes de material genético com espinho, originário do Pará e de Manaus, respectivamente.

A análise conjunta dos dados relativos dos quatro lotes (Figura 2) permitiu estimar a que redução de água os valores crítico e letal correspondem. Dessa forma, o teor de água crítico foi atingido quando houve perda igual ou superior a $40 \%$ do teor de água inicial das sementes. Perdas iguais ou acima de $70 \%$ do teor de água inicial das sementes levaram à letalidade.

Comprovou-se que sementes de pupunheira são recalcitrantes, com germinação inicial alta (59 a 84\%) quando não sujeitas à desidratação (47 a $38 \%$ de umidade inicial). Reduções significativas na germinação e vigor iniciam-se a partir de teores de umidade entre 28 e $23 \%$. Houve diferenças entre lotes em relação à germinação e ao vigor das sementes, e em relação à sensibilidade à desidratação, indicando que essas características são influenciadas pela colheita e manuseio das sementes, pelas condições agrobioclimáticas de cultivo e, possivelmente, componente genético.

\section{LITERATURA CITADA}

BECWAR, M.R.; STANWOOD, P.C.; ROSS, E.E. Dehydration effects imbibitional leakage from dessiccation sensitive seeds. Plant Physiology, v.69, n.4, p.1132-1135, 1982.

BOVI, M.L.A. Palmito pupunha: informações básicas para cultivo. Campinas, Instituto Agronômico, 1998. 50 p. (Boletim Técnico 173).

BOVI, M.L.A.; CHAVES FLORES, W.B.; SPIERING, S.H.; MARTINS, A.L.M.; PIZZINATTO, M.A.; LOURENÇÃO, A.L. Seed germination of progenies of Bactris gasipaes: porcentage, speed and duration. Acta Horticulturae, v.360, n.8, p.157-165, 1994.

CARVALHO, J.E.U.; MÜLLER, C.H. Níveis de tolerância e letal de umidade em sementes de pupunheira, Bactris gasipaes. Revista Brasileira de Fruticultura, v.20, n.3, p.283-289, 1998.

COATES-BECKFORD, P.L.; CHUNG, P.C. A study of the germination, disease symptoms and fungi associated with pejibaye seeds. Seed Science and Technology, v.15, n.1, p.205-218, 1987.

ELLIS, R.H.; HONG, T.D.; ROBERTS, E.H.; SOETISNA, U. Seed storage behaviour in Elaeis guineensis. Seed Science Research, v.1, n.1, p.99104, 1991.

FARRANT, J.M.; PAMMENTER, N.W.; BERJAK, P. Recalcitrance - a current assessment. Seed Science and Technology, v.16, n.1, p.155-166, 1988.

FERREIRA, S.A.N.; SANTOS L.A. Viabilidade de sementes de pupunha (Bactris gasipaes Kunth.). Acta Amazonica, v.22, n.3, p.303-307, 1992.

HONG, T.D.; ELLIS, R.H. Optimum air-dry seed storage environments for arabica coffee. Seed Science and Technology, v.20, n.3, p.547-560, 1992. MAGUIRE, J.D. Speed of germination aid in selection and evaluation for emergence and vigour. Crop Science, v.2, n.2, p.176-177, 1962.

MARTINS, C.C.; NAKAGAWA, J.; BOVI, M.L.A.; STANGUERLIM, H. Teores crítico e letal de água para sementes de açaizeiro (Euterpe oleracea Mart.). Revista Brasileira de Sementes, v.21, n.1, p.125-132, 1999a.

MARTINS, C.C.; NAKAGAWA, J.; BOVI, M.L.A. Desiccation tolerance of four seedlots from Euterpe edulis Mart. Seed Science and Technology, v.28, n.1, p.1-13, 1999 b.

MARTINS, C.C.; NAKAGAWA, J.; BOVI, M.L.A. Tolerância à dessecação de sementes de palmito-vermelho (Euterpe espiritosantensis Fernandes). Revista Brasileira de Botânica, v.22, n.3, p.391-396, 1999c.

PAMMENTER, N.W.; GREGGAINS, V.; KIOKO, J..I.; WESLEY-SMITH, J.; BERJAK, P.; FINCH-SAVAGE, W.E. Effects of differential drying rates on viability retention of recalcitrant seeds of Ekebergia capensis. Seed Science Research, v.8, n.4, p.463-471, 1998.

PROBERT, R.J.; LONGLEY, P.L. Recalcitrant seed storage physiology in three aquatic grasses (Zizania palustris, Spartina anglica and Portesia coarctata). Annals of Botany, v.63, n.1, p.53-63, 1989. 